\title{
The role of psychological interventions in the management of patients with chronic pain
}

This article was published in the following Dove Press journal:

Psychology Research and Behavior Management

10 May 2011

Number of times this article has been viewed

\author{
Daniela Roditi \\ Michael E Robinson \\ Department of Clinical and Health \\ Psychology, University of Florida, \\ Gainesville, FL, USA
}

\begin{abstract}
Chronic pain can be best understood from a biopsychosocial perspective through which pain is viewed as a complex, multifaceted experience emerging from the dynamic interplay of a patient's physiological state, thoughts, emotions, behaviors, and sociocultural influences. A biopsychosocial perspective focuses on viewing chronic pain as an illness rather than disease, thus recognizing that it is a subjective experience and that treatment approaches are aimed at the management, rather than the cure, of chronic pain. Current psychological approaches to the management of chronic pain include interventions that aim to achieve increased self-management, behavioral change, and cognitive change rather than directly eliminate the locus of pain. Benefits of including psychological treatments in multidisciplinary approaches to the management of chronic pain include, but are not limited to, increased self-management of pain, improved pain-coping resources, reduced pain-related disability, and reduced emotional distress - improvements that are effected via a variety of effective self-regulatory, behavioral, and cognitive techniques. Through implementation of these changes, psychologists can effectively help patients feel more in command of their pain control and enable them to live as normal a life as possible despite pain. Moreover, the skills learned through psychological interventions empower and enable patients to become active participants in the management of their illness and instill valuable skills that patients can employ throughout their lives.
\end{abstract}

Keywords: chronic pain management, psychology, multidisciplinary pain treatment, cognitive behavioral therapy for pain

\section{Introduction}

Pain is a ubiquitous human experience. It is estimated that approximately $20 \%-35 \%$ of adults experience chronic pain. ${ }^{1,2}$ The National Institute of Nursing Research reports that pain affects more Americans than diabetes, heart disease, and cancer combined. ${ }^{3}$ Pain has been cited as the primary reason to seek medical care in the United States. ${ }^{4}$ Furthermore, pain relievers are the second most commonly prescribed medications in physicians' offices and emergency rooms. ${ }^{5}$ Further solidifying the importance of adequate assessment of pain, the Joint Commission on the Accreditation of Healthcare Organizations issued a mandate requiring that pain be evaluated as the fifth vital sign during medical visits. ${ }^{6}$

The International Association for the Study of Pain (IASP) defines pain as "an unpleasant sensory and emotional experience associated with actual or potential tissue damage, or described in terms of such damage". 7 The IASP's definition highlights the multidimensional and subjective nature of pain, a complex experience that is unique to each individual. Chronic pain is typically differentiated from acute pain based on
Correspondence: Michael E Robinson Department of Clinical and Health Psychology, PO Box 100165, University of Florida, Gainesville, FL 32610-0165, USA

$\mathrm{Tel}+\mathrm{I} 3522736617$

Fax +I 3522736156

Email merobin@ufl.edu 
its chronicity or persistence, its physiological maintenance mechanisms, and/or its detrimental impact on an individual's life. Generally, it is accepted that pain that persists beyond the expected period of time for tissue healing following an injury or surgery is considered chronic pain. However, the specific timeframe constituting an expected healing period is variable and often difficult to ascertain. For ease of classification, certain guidelines suggest that pain persisting beyond a 3-6 month time window is considered chronic pain. ${ }^{7}$ Nevertheless, classification of pain based solely on duration is a strictly practical and, in some instances, arbitrary criterion. More commonly, additional factors such as etiology, pain intensity, and impact are considered alongside duration when classifying chronic pain. An alternative way to characterize chronic pain has been based on its physiological maintenance mechanism; that is, pain that is thought to emerge as a result of peripheral and central reorganization. Common chronic pain conditions include musculoskeletal disorders, neuropathic pain conditions, headache pain, cancer pain, and visceral pain. More broadly, pain conditions may be primarily nociceptive (producing mechanical or chemical pain), neuropathic (resulting from nerve damage), or central (resulting from dysfunction in the neurons of the central nervous system). ${ }^{8}$

Unfortunately, the experience of pain is frequently characterized by undue physical, psychological, social, and financial suffering. Chronic pain has been recognized as the leading cause of long-term disability in the working-age American population. ${ }^{9}$ Because chronic pain affects the individual at multiple domains of his/her existence it also constitutes an enormous financial burden to our society. The combined direct and indirect costs of pain have been estimated to range from $\$ 125$ billion to $\$ 215$ billion, annually. ${ }^{10,11}$ The widespread implications of chronic pain include increased reports of emotional distress (eg, depression, anxiety, and frustration), increased rates of pain-related disability, pain-related alterations in cognition, and reduced quality of life. Thus, chronic pain can be best understood from a biopsychosocial perspective through which pain is viewed as a complex, multifaceted experience emerging from the dynamic interplay of a patient's physiological state, thoughts, emotions, behaviors, and sociocultural influences.

\section{Pain management}

Given the widespread prevalence of pain and its multidimensional nature, an ideal pain management regimen will be comprehensive, integrative, and interdisciplinary. Current approaches to the management of chronic pain have increasingly transcended the reductionist and strictly surgical, physical, or pharmacological approach to treatment. Current approaches recognize the value of a multidisciplinary treatment framework that targets not only nociceptive aspects of pain but also cognitive-evaluative, and motivationalaffective aspects alongside equally unpleasant and impacting sequelae. The interdisciplinary management of chronic pain typically includes multimodal treatments such as combinations of analgesics, physical therapy, behavioral therapy, and psychological therapy. The multimodal approach more adequately and comprehensively addresses pain management at the molecular, behavioral, cognitive-affective, and functional levels. These approaches have been shown to lead to superior and long-lasting subjective and objective outcomes including pain reports, mood, restoration of daily functioning, work status, and medication or health care use; multimodal approaches have also been shown to be more cost-effective than unimodal approaches. ${ }^{12,13}$ The focus of this review will be specifically on elucidating the benefits of psychology in the management of chronic pain.

Patients will typically initially present to a physician's office in the pursuit of a cure or treatment for their ailment/ acute pain. For many patients, depending on the etiology and pathology of their pain alongside biopsychosocial influences on the pain experience, acute pain will resolve with the passage of time, or following treatments aimed at targeting the presumed cause of pain or its transmission. Nonetheless, some patients will not achieve resolution of their pain despite numerous medical and complementary interventions and will transition from an acute pain state to a state of chronic, intractable pain. For instance, research has demonstrated that approximately $30 \%$ of patients presenting to their primarycare physician for complaints related to acute back pain will continue to experience pain and, for many others, severe activity limitations and suffering 12 months later. ${ }^{14}$ As pain and its consequences continue to develop and manifest in diverse aspects of life, chronic pain may become primarily a biopsychosocial problem, whereby numerous biopsychosocial aspects may serve to perpetuate and maintain pain, thus continuing to negatively impact the affected individual's life. It is at this point that the original treatment regimen may diversify to include other therapeutic components, including psychological approaches to pain management.

Psychological approaches for the management of chronic pain initially gained popularity in the late $1960 \mathrm{~s}$ with the emergence of Melzack and Wall's "gate-control theory of pain" 15 and the subsequent "neuromatrix theory of pain". ${ }^{16}$ Briefly, these theories posit that psychosocial and physiological processes interact to affect perception, 
transmission, and evaluation of pain, and recognize the influence of these processes as maintenance factors involved in the states of chronic or prolonged pain. Namely, these theories served as integral catalysts for instituting change in the dominant and unimodal approach to the treatment of pain, one heavily dominated by strictly biological perspectives. Clinicians and patients alike gained an increasing recognition and appreciation for the complexity of pain processing and maintenance; consequently, the acceptance of and preference for multidimensional conceptualizations of pain were established. Currently, the biopsychosocial model of pain is, perhaps, the most widely accepted heuristic approach to understanding pain. ${ }^{17} \mathrm{~A}$ biopsychosocial perspective focuses on viewing chronic pain as an illness rather than disease, thus recognizing that it is a subjective experience and that treatment approaches are aimed at the management, rather than the cure, of chronic pain. ${ }^{17}$ As the utility of a broader and more comprehensive approach to the management of chronic pain has become evident, psychologically-based interventions have witnessed a remarkable rise in popularity and recognition as adjunct treatments. The types of psychological interventions employed as part of a multidisciplinary pain treatment program vary according to therapist orientation, pain etiology, and patient characteristics. Likewise, research on the effectiveness of psychologically based interventions for chronic pain has shown variable, albeit promising, results on key variables studied. This overview will briefly describe frequently employed psychologically based treatment options and their respective effectiveness on key outcomes.

Current psychological approaches to the management of chronic pain include interventions that aim to achieve increased self-management, behavioral change, and cognitive change rather than directly eliminate the locus of pain. As such, they target the frequently overlooked behavioral, emotional, and cognitive components of chronic pain and factors contributing to its maintenance. Informed by the framework offered by Hoffman et $\mathrm{al}^{18}$ and Kerns et al, ${ }^{19}$ the following frequently employed psychologically-based treatment domains are reviewed: psychophysiological techniques, behavioral approaches to treatment, cognitive behavioral therapy, and acceptance-based interventions.

\section{Psychophysiological techniques Biofeedback}

Biofeedback is a learning technique through which patients learn to interpret feedback (in the form of physiological data) regarding certain physiological functions. For instance, a patient may use biofeedback equipment to learn to recognize areas of tension in their body and subsequently learn to relax those areas to reduce muscular tension. Feedback is provided by a variety of measurement instruments that can yield information about brain electrical activity, blood pressure, blood flow, muscle tone, electrodermal activity, heart rate, and skin temperature, among other physiological functions in a rapid manner. The goal of biofeedback approaches is for the patient to learn how to initiate physiological self-regulatory processes by achieving voluntary control over certain physiological responses to ultimately increase physiological flexibility through greater awareness and specific training. Thus a patient will use specific self-regulatory skills in an attempt to reduce an undesired event (eg, pain) or maladaptive physiological reactions to an undesired event (eg, stress response). Many psychologists are trained in biofeedback techniques and provide these services as part of therapy. Biofeedback has been designated as an efficacious treatment for pain associated with headache and temporomandibular disorders (TMD) ${ }^{20}$ A meta-analysis of 55 studies revealed that biofeedback interventions (including various biofeedback modalities) yielded significant improvements with regard to frequency of migraine attacks and perceptions of headache management self-efficacy when compared to control conditions. ${ }^{21}$ Studies have provided empirical support for biofeedback for TMD, albeit more robust improvements with regard to pain and pain-related disability have been found for protocols that combine biofeedback with cognitive behavioral skills training, under the assumption that a combined treatment approach more comprehensively addresses the gamut of biopsychosocial problems that may be encountered as a result of TMD. ${ }^{22}$

\section{Behavioral approaches Relaxation training}

It is generally accepted that stress is a key factor involved in the exacerbation and maintenance of chronic pain. ${ }^{16,23}$ Stress can be predominantly of an environmental, physical, or psychological/emotional basis, though typically these mechanisms are intricately intertwined. The focus of relaxation training is to reduce tension levels (physical and mental) through activation of the parasympathetic nervous system and through attainment of greater awareness of physiological and psychological states, thereby achieving reductions in pain and increasing control over pain. Patients can be taught several relaxation techniques and practice them individually or in conjunction with one another, as well as adjuvant components to other behavioral and cognitive pain management techniques. The following are brief descriptions 
of relaxation techniques commonly taught by psychologists specializing in the management of chronic pain.

\section{Diaphragmatic breathing}

Diaphragmatic breathing is a basic relaxation technique whereby patients are instructed to use the muscles of their diaphragm as opposed to the muscles of their chest to engage in deep breathing exercises. Breathing by contracting the diaphragm allows the lungs to expand down (marked by expansion of abdomen during inhalation) and thus increase oxygen intake. ${ }^{24}$

\section{Progressive muscle relaxation (PMR)}

PMR is characterized by engaging in a combination of muscle tension and relaxation exercises of specific muscles or muscle groups throughout the body. ${ }^{25}$ The patient is typically instructed to engage in the tension/relaxation exercises in a sequential manner until all areas of the body have been addressed.

\section{Autogenic training (AT)}

AT is a self-regulatory relaxation technique in which a patient repeats a phrase in conjunction with visualization to induce a state of relaxation. ${ }^{26,27}$ This method combines passive concentration, visualization, and deep breathing techniques.

\section{Visualization/Guided imagery}

This technique encourages patients to use all of their senses in imagining a vivid, serene, and safe environment to achieve a sense of relaxation and distraction from their pain and pain-related thoughts and sensations. ${ }^{27}$

Collectively, relaxation techniques have generally been found to be beneficial in the management of a variety of types of acute and chronic pain conditions as well as in the management of important pain sequelae (eg, health-related quality of life). ${ }^{28-31}$ Relaxation techniques are usually practiced in conjunction with other pain management modalities, and there is considerable overlap in the presumed mechanisms of relaxation and biofeedback, for instance.

\section{Operant behavior therapy}

Operant behavior therapy for chronic pain is guided by the original operant conditioning principles proposed by Skinner $^{32}$ and refined by Fordyce ${ }^{33}$ to be applicable to pain management. The main tenets of the operant conditioning model as it relates to pain hold that pain behavior can eventually evolve into and be maintained as chronic pain manifestations as a result of positive or negative reinforcement of a given pain behavior as well as punishment of more adaptive, non-pain behavior. If reinforcement and the ensuing consequences occur with sufficient frequency, they can serve to condition the behavior, thus increasing the likelihood of repeating the behavior in the future. Therefore, conditioned behaviors occur as a product of learning of the consequences (actual or anticipated) of engaging in the given behavior. An example of a conditioned behavior is continued use of medication - a behavior that results from learning through repeated associations that taking medication is followed by removal of an aversive sensation (pain). Likewise, pain behaviors (eg, verbal expressions of pain, low activity levels) can be become conditioned behaviors that serve to perpetuate chronic pain and its sequelae. Treatments that are guided by operant behavior principles aim to extinguish maladaptive pain behaviors through the same learning principles that these may have been established by. In general, treatment components of operant behavior therapy include graded activation, time contingent medication schedules, and use of reinforcement principles to increase well behaviors and decrease maladaptive pain behaviors.

\section{Graded activation}

Psychologists can implement graded activity programs for chronic pain patients who have vastly reduced their activity levels (increasing likelihood of physical deconditioning) and subsequently experience high levels of pain upon engaging in activity. Patients are instructed to safely break the cycle of inactivity and deconditioning by engaging in activity in a controlled and time-limited fashion. In this manner, patients can gradually increase the length of time and intensity of activity to improve functioning. Psychologists can oversee progress and provide appropriate reinforcement for compliance, correction of misperceptions or misinterpretations of pain resulting from activity, where appropriate, and problem-solve barriers to adherence. This approach is frequently embedded within cognitive-behavioral pain management treatments.

\section{Time-contingent medication schedules}

A psychologist can be an important adjunct healthcare provider in overseeing the management of pain medications. In some cases, psychologists have the opportunity for more frequent and in-depth contact with patients than physicians and thus can serve as valuable collaborators of an integrated multidisciplinary treatment approach. Psychologists can institute time-contingent medication schedules to reduce the likelihood of dependence on pain medications for attaining adequate control over pain. Furthermore, psychologists are well equipped to engage patients in important conversations 
regarding the importance of proper adherence to medications and medical recommendations and problem-solve perceived barriers to safe adherence.

\section{Fear-avoidance}

The fear-avoidance model of chronic pain is a heuristic most frequently applied in the context of chronic low back pain (LBP).${ }^{34}$ This model draws largely from the operant behavior principles described previously. In essence, the fear-avoidance model posits that when acute pain states are repeatedly misinterpreted as danger signals or signs of serious injury, patients may be at risk of engaging in fear-driven avoidance behaviors and cognitions that further reinforce the belief that pain is a danger signal and perpetuate physical deconditioning. As the cycle continues, avoidance may generalize to broader types of activity and result in hypervigilance of physical sensations characterized by misinformed catastrophic interpretations of physical sensations. Research has shown that a high degree of pain catastrophizing is associated with maintenance of the cycle. ${ }^{35}$ Treatments aimed at breaking the fear-avoidance cycle employ systematic graded exposure to feared activities to disconfirm the feared, often catastrophic, consequences of engaging in activities. Graded exposure is typically supplemented with psychoeducation about pain and cognitive restructuring elements that target maladaptive cognitions and expectations about activity and pain. Psychologists are in an excellent position to execute these types of interventions that closely mimic exposure treatments traditionally used in the treatment of some anxiety disorders.

Though specific graded exposure treatments have been shown to be effective in the treatment of complex regional pain syndrome type I (CRPS-1) ${ }^{36}$ and $\mathrm{LBP}^{37}$ in single-case designs, a larger-scale randomized controlled trial comparing systematic graded exposure treatment combined with multidisciplinary pain program treatment with multidisciplinary pain program treatment alone and with a wait-list control group found that the two active treatments resulted in significant improvements on outcome measures of pain intensity, fear of movement/injury, pain self-efficacy, depression, and activity level. ${ }^{38}$ Results from this trial suggest that both interventions were associated with significant treatment effectiveness such that the graded exposure treatment did not appear to result in additional treatment gains. ${ }^{38}$ A cautionary note in the interpretation of these results highlights that the randomized controlled trial (RCT) included a variety of chronic pain conditions that extended beyond LBP and CRPS-1 and did not exclusively include patients with high levels of pain-related fear; the interventions were also delivered in group formats rather than individual formats. Although in-vivo exposure treatments are superior at reducing pain catastrophizing and perceptions of harmfulness of activities, exposure treatments seem to be as effective as graded activity interventions in improving functional disability and chief complaints. ${ }^{39}$ Another clinical trial compared the effectiveness of treatment-based classification (TBC) physical therapy alone to TBC augmented with graded activity or graded exposure for patients with acute and sub-acute LBP. ${ }^{40}$ Outcomes revealed that there were no differences in 4-week and 6-month outcomes for reduction of disability, pain intensity, pain catastrophizing, and physical impairment among treatment groups, although graded exposure and TBC yielded larger reductions in fear-avoidance beliefs at 6 months. ${ }^{40}$ Findings from this clinical trial suggest that enhancing TBC with graded activity or graded exposure does not lead to improved outcomes with regard to measures associated with the development of chronic LBP beyond improvements achieved with $\mathrm{TBC}$ alone. ${ }^{40}$

\section{Cognitive-behavioral approaches}

Cognitive-behavioral therapy (CBT) interventions for chronic pain utilize psychological principles to effect adaptive changes in the patient's behaviors, cognitions or evaluations, and emotions. These interventions are generally comprised of basic psychoeducation about pain and the patient's particular pain syndrome, several behavioral components, coping skills training, problem-solving approaches, and a cognitive restructuring component, though the exact treatment components vary according to the clinician. Behavioral components may include a variety of relaxation skills (as reviewed in the behavioral approaches section), activity pacing instructions/graded activation, behavioral activation strategies, and promotion of resumption of physical activity if there is a significant history of activity avoidance and subsequent deconditioning. The primary aim in coping skills training is to identify current maladaptive coping strategies (eg, catastrophizing, avoidance) that the patient is engaging in alongside their use of adaptive coping strategies (eg, use of positive self-statements, social support). As a cautionary note, the degree to which a strategy is adaptive or maladaptive and the perceived effectiveness of particular coping strategies varies from individual to individual. ${ }^{41}$ Throughout treatment, problem-solving techniques are honed to aid patients in their adherence efforts and to help them increase their self-efficacy. Cognitive restructuring entails recognition of current maladaptive cognitions the patient is engaging in, challenging of the identified negative cognitions, and reformulation of 
thoughts to generate balanced, adaptive alternative thoughts. Through cognitive restructuring exercises, patients become increasingly adept at recognizing how their emotions, cognitions, and interpretations modulate their pain in positive and negative directions. As a result, it is presumed that the patients will attain a greater perception of control over their pain, be better able to manage their behavior and thoughts as they relate to pain, and be able to more adaptively evaluate the meaning they ascribe to their pain. Additional components sometimes included in a CBT intervention include social skills training, communication training, and broader approaches to stress management. Via a pain-oriented CBT intervention, many patients profit from improvements with regard to their emotional and functional well-being, and ultimately their global perceived health-related quality of life.

CBT interventions are delivered within a supportive and empathetic environment that strives to understand the patient's pain from a biopsychosocial perspective and in an integrated manner. Therapists see their role as "teachers" or "coaches" and the message communicated to patients is that of learning to better manage their pain and improve their daily function and quality of life as opposed to aiming to cure or eradicate the pain. The overarching goal is to increase the patients' understanding of their pain and their efforts to manage pain and its sequelae in a safe and adaptive manner; therefore, teaching patients to self-monitor their behavior, thoughts, and emotions is an integral component of therapy and a useful strategy to enhance self-efficacy. Additionally, the therapist endeavors to foster an optimistic, realistic, and encouraging environment in which the patient can become increasingly skilled at recognizing and learning from their successes and learning from and improving upon unsuccessful attempts. In this manner, therapists and patients work together to identify patient successes, barriers to adherence, and to develop maintenance and relapse-prevention plans in a constructive, collaborative, and trustworthy atmosphere. An appealing feature of the cognitive behavioral approach is its endorsement of the patient as an active participant of his/her pain rehabilitation or management program.

Research has found CBT to be an effective treatment for chronic pain and its sequelae as marked by significant changes in various domains (ie, measures of pain experience, mood/ affect, cognitive coping and appraisal, pain behavior and activity level, and social role function) when compared with waitlist control conditions. ${ }^{42}$ When compared with other active treatments or control conditions, CBT has resulted in notable improvements, albeit smaller effects (effect size $\sim 0.50$ ), with regard to pain experience, cognitive coping and appraisal, and social role function. ${ }^{42} \mathrm{~A}$ more recent meta-analysis of 52 published studies compared behavior therapy (BT) and CBT against treatment as usual control conditions and active control conditions at various time-points. ${ }^{43}$ This meta-analysis concluded that their data did not lend support for BT beyond improvements in pain immediately following treatment when compared with treatment as usual control conditions. ${ }^{43}$ With regard to CBT, they concluded that CBT has limited positive effects for pain disability, and mood; nonetheless, there are insufficient data available to investigate the specific influence of treatment content on selected outcomes. ${ }^{43}$ Overall, it appears that CBT and BT are effective treatment approaches to improve mood; outcomes that remain robust at follow-up data points. However, as highlighted by several reviews and meta-analyses, a critical factor to consider in evaluating the effectiveness of CBT for the management of chronic pain is centered on issues of effective delivery, lack of uniform treatment components, differences in delivery across clinicians and treatment populations, and variability in outcome variables of interest across research trials. ${ }^{13}$ Further complicating the interpretation of effectiveness findings are patient characteristics and additional variables that may independently affect treatment outcome.

\section{Acceptance-based approaches}

Acceptance-based approaches are frequently identified as third-wave cognitive-behavioral therapies. Acceptance and commitment therapy (ACT) is the most common of the acceptance-based psychotherapies. ACT emphasizes the importance of facilitating the client's progress toward attaining a more valued and fulfilling life by increasing psychological flexibility rather than strictly focusing on restructuring cognitions. ${ }^{44}$ In the context of chronic pain, ACT targets ineffective control strategies and experiential avoidance by fostering techniques that establish psychological flexibility. The six core processes of ACT include: acceptance, cognitive defusion, being present, self as context, values, and committed action. ${ }^{45}$ Briefly, acceptance encourages chronic pain patients to actively embrace pain and its sequelae rather than attempt to change it, in doing so encouraging the patient to cease a futile fight directed at the eradication of their pain. Cognitive defusion (deliteralization) techniques are employed to modify the function of thoughts rather than to reduce their frequency or restructure their content. In this manner, cognitive defusion may simply alter the undesirable meaning or function of negative thoughts and thus decrease the attachment and subsequent emotional and behavioral response to such thoughts. The core process of being present 
emphasizes a non-judgmental interaction between the self and private thoughts and events. Values are utilized as guides for electing behaviors and interpretations that are characterized by those values an individual strives to instantiate in everyday life. Finally, through committed action, patients can realize behavior changes aligned with individual values. Thus, ACT utilizes the six core principles in conjunction with one another to take a holistic approach toward increasing psychological flexibility and decreasing suffering. Patients are encouraged to view pain as inevitable and accept it in a nonjudgmental manner so that they can continue to derive meaning from life despite the presence of pain. The interrelated core processes exemplify mindfulness and acceptance processes and commitment and behavior change processes. ${ }^{45}$

Results of research on the effectiveness of ACT-based approaches for the management of chronic pain are promising, albeit still warranting further evaluation. A RCT comparing ACT with a waitlist control condition reported significant improvements in pain catastrophizing, painrelated disability, life satisfaction, fear of movements, and psychological distress that were maintained at the 7 month follow-up. ${ }^{46} \mathrm{~A}$ larger trial reported significant improvements for pain, depression, pain-related anxiety, disability, medical visits, work status, and physical performance. ${ }^{47} \mathrm{~A}$ recent meta-analysis evaluating acceptance-based interventions (ACT and mindfulness-based stress reduction) in patients with chronic pain found that, in general, acceptance-based therapies lead to favorable outcomes for patients with chronic pain. ${ }^{48}$ Specifically, the meta-analysis revealed small to medium effect sizes for pain intensity, depression, anxiety, physical wellbeing, and quality of life, with smaller effects found when controlled clinical trials were excluded and only RCTs were included in the analyses. ${ }^{48}$ Other acceptancebased interventions include contextual cognitive-behavioral therapy and mindfulness-based cognitive therapy, though empirical research on the effectiveness of these therapies for the management of chronic pain is still in its infancy.

\section{Expectations}

An important and vastly overlooked common underlying element of all treatment approaches is consideration of the patient's expectation for treatment success. Despite the numerous advances in the formulation and delivery of effective multidisciplinary treatments for chronic pain, relatively little emphasis has been placed on recognizing the importance of expectations for success and on focusing efforts on enhancement of patients' expectations. The recognition that placebo for pain is characterized by active properties leading to reliable, observable, and quantifiable changes with neurobiological underpinnings is currently at the vanguard of pain research. Numerous studies have confirmed that, when induced in a manner that optimizes expectations (via manipulation of explicit expectations and/or conditioning), analgesic placebos can result in observable and measurable changes in pain perception at a conscious self-reported level as well as a neurological pain-processing level. ${ }^{49,50}$ Analgesic placebos have been broadly defined as simulated treatments or procedures that occur within a psychosocial context and exert effects on an individual's experience and/or physiology. ${ }^{51}$ The current conceptualization of placebo emphasizes the importance of the psychosocial context within which placebos are embedded. Underlying the psychosocial context and ritual of treatment are patients' expectations. Therefore, it is not surprising that the placebo effect is intricately embedded in virtually every treatment; as such, clinicians and patients alike will likely benefit from recognition that therein lies an additional avenue by which current treatment approaches to pain can be enhanced.

It has been proposed that outcome expectancies are core influences driving the positive changes attained through the various modes of relaxation training, hypnosis, exposure treatments, and many cognitive-oriented therapeutic approaches. Thus, a sensible approach to the management of chronic pain capitalizes on the power of patients' expectations for success. Regrettably, too often, health care providers neglect to directly address and emphasize the importance of patients' expectations as integral factors contributing to successful management of chronic pain. The zeitgeist in our society is that of mounting medicalization of ailments fueling the general expectation that pain (even chronic pain) ought to be eradicated through medical advancements. These all too commonly held expectations leave many patients disillusioned with current treatment outcomes and contribute to an incessant search for the "cure". Finding the "cure" is the exception rather than the rule with respect to chronic pain conditions. In our current climate, where chronic pain afflicts millions of Americans annually, it is in our best interest to instill and continue to advocate a conceptual shift that instead focuses on effective management of chronic pain. A viable and promising route to achieving this is to make the most of patients' positive (realistic) expectations and educate pain patients as well as the lay public ( $20 \%$ of whom will at some future point become pain patients) on what constitutes realistic expectations regarding the management of pain. Perhaps, this can occur initially through current, evidence-based 
education regarding placebo and nonspecific treatment effects such that patients can correct misinformed beliefs they may have previously held. Subsequently clinicians can aim to enhance patients' expectations within treatment contexts (in a realistic fashion) and minimize pessimistic expectations that deter from treatment success, therefore, learning to enhance their current multidisciplinary treatments through efforts guided at capitalizing on the improvements placebo can yield, even within an "active treatment". Psychologists can readily address these issues with their patients and help them become advocates of their own treatment success.

\section{Emotional concomitants of pain}

An often challenging aspect of the management of chronic pain is the unequivocally high prevalence of comorbid emotional distress. Research has demonstrated that depression and anxiety disorders are upward to three times more prevalent among chronic pain patients than among the general population. ${ }^{52,53}$ Frequently, pain patients with psychiatric comorbidities are labeled "difficult patients" by healthcare providers, possibly diminishing the quality of care they will receive. Patients with depression have poorer outcomes for both depression and pain treatments, compared with patients with single diagnoses of pain or depression. ${ }^{54,55}$ Psychologists are remarkably suited to address most of the psychiatric comorbidities typically encountered in chronic pain populations and thus improve pain treatment outcomes and decrease the emotional suffering of patients. Psychologists can address key symptoms (eg, anhedonia, low motivation, problemsolving barriers) of depression that readily interfere with treatment participation and emotional distress. Moreover, irrespective of a psychiatric comorbidity, psychologists can help chronic pain patients process important role transitions they may undergo (eg, loss of job, disability), interpersonal difficulties they may be encountering (eg, sense of isolation brought about by pain), and emotional suffering (eg, anxiety, anger, sadness, disappointment) implicated in their experience. Thus, psychologists can positively impact the treatment course by reducing the influence of emotional concomitants that are addressed as part of therapy.

\section{Conclusion}

Benefits of including psychological treatments in multidisciplinary approaches to the management of chronic pain are abundant. These include, but are not limited to, increased self-management of pain, improved pain-coping resources, reduced pain-related disability, and reduced emotional distress-improvements that are effected via a variety of effective self-regulatory, behavioral, and cognitive techniques. Through implementation of these changes, a psychologist can effectively help patients feel more in command of their pain control and enable them to live as normal a life as possible despite pain. Moreover, the skills learned through psychological interventions empower and enable patients to become active participants in the management of their illness and instill valuable skills that patients can employ throughout their lives. Additional benefits of an integrated and holistic approach to the management of chronic pain may include increased rates of return to work, reductions in health care costs, and increased health-related quality of life for millions of patients throughout the world.

\section{Disclosure}

No conflicts of interest were declared in relation to this paper.

\section{References}

1. Boris-Karpel S. Policy and practice issues in pain management. In: Ebert MH, Kerns RD, editors. Behavioral and psychopharmacologic pain management. New York: Cambridge University Press; 2010:407-433.

2. Harstall C, Ospina M. How prevalent is chronic pain? Pain: Clinical Updates. 2003;11(2):1-4.

3. National Institutes of Health. Fact sheet: pain management. Available from: http://www.ninr.nih.gov/NR/rdonlyres/DC0351 A6-7029-4FE0BEEA-7EFC3D1B23AE/0/Pain.pdf (2007). Accessed 30 Mar 2011.

4. Abbot FV, Fraser MI. Use and abuse of over-the-counter analgesic agents. J Psychiatry Neurosci. 1998;23(1):13-34.

5. Schappert SM, Burt CW. Ambulatory care visits to physician offices, hospital outpatient departments, and emergency departments: United States, 2001-02. Vital Health Stat. 2006;13(159):1-66.

6. Joint Commission of Accreditation of Healthcare Organizations. Pain assessment and management: an organizational approach. Oakbrook, IL; 2000.

7. Task Force on Taxonomy of the IASP. Part III: Pain terms, a current list with definitions and notes on usage. In: Merskey H, Bogduk N, editors. Classification of chronic pain. 2nd edition. Seattle, WA: IASP Press; 1994:209-214.

8. Woessner J. A conceptual model of pain: treatment modalities. Pract Pain Manag. 2003;3(1)26-36.

9. Loeser JD. Economic implications of pain management. Acta Anaesthesiol Scand. 1999;43(9):957-959.

10. National Research Council. Musculoskeletal disorders and the workplace: low back and upper extremities. Washington, DC: National Academy Press; 2001.

11. US Bureau of the Census. Statistical abstract of the United States: 1996. 116th edition. Washington, DC.

12. Flor H, Fydrich T, Turk DC. Efficacy of multidisciplinary pain treatment centers: a meta-analytic review. Pain. 1992;49(2):221-230.

13. McCracken LM, Turk DC. Behavioral and cognitive-behavioral treatment for chronic pain: outcome, predictors of outcome, and treatment process. Spine. 2002;27(22):2564-2573.

14. Von Korff M, Saunders K. The course of back pain in primary care. Spine. 1996;21(24):2833-2837.

15. Melzack R, Wall PD. Pain mechanisms: a new theory. Science. 1965;150(699):971-979.

16. Melzack R. Pain and stress: a new perspective. In: Gatchel RJ, Turk DC, editors. Psychosocial factors in pain: critical perspectives. New York: Guilford Press; 1999:89-106. 
17. Gatchel RJ. The conceptual foundations of pain management: historical overview. In: Gatchel RJ, editor. Clinical essentials of pain management Washington, DC: American Psychological Association; 2005:3-16.

18. Hoffman BM, Papas RK, Chatkoff DK, Kerns RD. Meta-analysis of psychological interventions for chronic low back pain. Health Psychol. 2007;26(1):1-9.

19. Kerns RD, Sellinger J, Goodin BR. Psychological treatment of chronic pain. Annu Rev Clin Psychol. 2010 Sep 27. [Epub ahead of print]

20. Yucha C, Montgomery D. Evidence-based practice in biofeedback and neurofeedback. Wheat Ridge, CO: AAPB; 2008.

21. Nestoriuc Y, Martin A. Efficacy of biofeedback for migraine: a meta-analysis. Pain. 2007;128(1-2):111-127.

22. Gardea MA, Gatchel RJ, Mishra KD. Long-term efficacy of biobehavioral treatment of temporomandibular disorders. J Behav Med 2001;24(4):341-359.

23. Turk DC, Monarch ES. Biopsychosocial perspective on chronic pain. In: Turk DC, Gatchel RJ, editors. Psychosocial approaches to pain management: a practitioner's handbook. 2nd edition. New York Guilford Press; 2002:3-29.

24. Philips HC. Orientation: chronic pain and the self-management approach. In: The psychological management of chronic pain: a treatment manual. New York: Springer Publishing; 1988:45-60.

25. Bernstein DA, Borkovek TD. Progressive muscle relaxation training. a manual for helping professions. Champaign, IL: Research Press; 1973.

26. Linden W. Autogenic training: a clinical guide. New York: Guilford; 1990.

27. Jamison RN. Mastering chronic pain: a professional's guide to behavioral treatment. Sarasota, FL: Professional Resource Press; 1996.

28. Baird CL, Sands L. Effect of guided imagery with relaxation on health-related quality of life in older women with osteoarthritis. Res Nurs Health. 2006;29(5):442-451.

29. Carroll D, Seers K. Relaxation for the relief of chronic pain: a systematic review. J Adv Nurs. 1998;27(3):476-487.

30. Morone NE, Greco CM. Mind-body interventions for chronic pain in older adults: a structured review. Pain Med. 2007;8(4):359-375.

31. Mannix LK, Chandurkar RS, Rybicki LA, Tusek DL, Solomon GD. Effect of guided imagery on quality of life for patients with chronic tension-type headache. Headache. 1999;39(5):326-334.

32. Skinner BF. Science and human behavior. New York: Free Press; 1953.

33. Fordyce WE. Behavioural methods for chronic pain and illness. London, UK: The CV Mosby Company; 1976.

34. Vlayen JW, Linton SJ. Fear-avoidance and its consequences in chronic musculoskeletal pain: a state of the art. Pain. 2000;85(3):317-332.

35. Vlayen JW, de Jong J, Sieben J, Crombez G. Graded exposure in vivo for pain-related fear. In: Turk DC, Gatchel RJ, editors. Psychosocial approaches to pain management: a practitioner's handbook. 2nd edition. New York: Guilford Press; 2002:210-233.

36. De Jong JR, Vlaeyen JW, Onghena P, Cuypers C, den Hollander M, Ruijgrok J. Reduction of pain-related fear in complex regional pain syndrome type I: the application of graded exposure in vivo. Pain. 2005;116(3): 264-275.

37. Boersma K, Linton S, Overmeer T, Jansson M, Vlaeyen J, de Jong J. Lowering fear-avoidance and enhancing function through exposure in vivo: a multiple baseline study across six patients with back pain. Pain. 2004;108(1-2):8-16.
38. Bliokas VV, Cartmill TK, Nagy BJ. Does systematic graded exposure in vivo enhance outcomes in multidisciplinary chronic pain management groups? Clin J Pain. 2007;23(4):361-374.

39. Leeuw M, Goossens ME, van Breukelen GJ, et al. Exposure in vivo versus operant graded activity in chronic low back pain patients: results of a randomized controlled trial. Pain. 2008;138(1):192-207.

40. George SZ, Zeppieri G, Cere AL, et al. A randomized trial of behavioral physical therapy interventions for acute and sub-acute low back pain (NCT00373867). Pain. 2008;140(1):145-157.

41. Roditi D, Waxenberg LB, Robinson ME. Frequency and perceived effectiveness of coping define important subgroups of patients with chronic pain. Clin J Pain. 2010;26(8):677-682.

42. Morley S, Eccleston C, Williams A. Systematic review and metaanalysis of randomized controlled trials of cognitive behaviour therapy and behaviour therapy for chronic pain in adults, excluding headache. Pain. 1999;80(1-2):1-13.

43. Eccleston C, Williams AC, Morley S. Psychological therapies for the management of chronic pain (excluding headache) in adults. Cochrane Database Syst Rev. 2009;(2):CD007407.

44. Blackledge JT, Hayes SC. Emotion regulation in acceptance and commitment therapy. J Clin Psychol. 2001;57(2):243-255.

45. Hayes SC, Luoma JB, Bond FW, Masuda A, Lillis J. Acceptance and commitment therapy: model, processes, and outcomes. Behav Res Ther. 2006;44(1):1-25.

46. Wicksell RK, Ahlqvist J, Bring A, Melin L, Olsson GL. Can exposure strategies improve functioning and life satisfaction in people with chronic pain and whiplash-associated disorders (WAD)? A randomized controlled trial. Cogn Behav Ther. 2008;37(3):169-182.

47. Vowles KE, McCracken LM. Acceptance and values-based action in chronic pain: a study of treatment effectiveness and process. $J$ Consult Clinl Psychol. 2008;76(3):397-407.

48. Veehof MM, Oskam MJ, Schreurs KMG, Bohlmeijer ET. Acceptancebased interventions for the treatment of chronic pain: a systematic review and meta-analysis. Pain. 2011;152(3):533-542.

49. Wager TD, Rilling JK, Smith EE, et al. Placebo-induced changes in $f$ MRI in the anticipation and experience of pain. Science. 2004;303(5661): 1162-1167.

50. Price DD, Craggs J, Verne GN, Perlstein WM, Robinson ME. Placebo analgesia is accompanied by large reductions in pain-related brain activity in irritable-bowel syndrome patients. Pain. 2007;127(1-2):63-72.

51. Price D, Finniss D, Benedetti F. A comprehensive review of the placebo effect: recent advances and current thought. Annu Rev Psychol. 2008;59:565-590.

52. Holroyd KA. Recurrent headache disorders. In: Dworkin RH, Breitbart WS, editors. Psychosocial aspects of pain: a handbook for health care providers. Seattle, WA: IASP Press; 2004:370-403.

53. Fishbain DA. Approaches to treatment decisions for psychiatric comorbitity in the management of the chronic pain patient. Med Clin North Am. 1999;83(3):737-760.

54. Bair MJ, Robinson RL, Katon W, Kroenke K. Depression and pain comorbidity - a literature review. Arch Intern Med. 2003;163(20): 2433-2445.

55. Poleshuck EL, Talbot NL, Su H, et al. Pain as a predictor of depression treatment outcomes in women with childhood sexual abuse. Compr Psychiatry. 2009;50(3):215-220.

Psychology Research and Behavior Management

\section{Publish your work in this journal}

Psychology Research and Behavior Management is an international, peerreviewed, open access journal focusing on the science of psychology and its application in behavior management to develop improved outcomes in the clinical, educational, sports and business arenas. Specific topics covered include: Neuroscience, memory \& decision making; Behavior

modification \& management; Clinical applications; Business \& sports performance management; Social and developmental studies; Animal studies. The manuscript management system is completely online and includes a quick and fair peer-review system. Visit http://www.dovepress. com/testimonials.php to read real quotes from published authors. 